\title{
How man fabricates nano-sized objects
}

\author{
Anushka Gangnaik \\ Chemistry Department, UCC
}

"There's Plenty of Room at the Bottom." (Richard Feynman)

\section{Introduction to nanoworld}

In this fast paced world we are constantly surrounded by novel and amusing technologies. The Apple Macbook is one of the modern devices that is used by many people. Not only has the technology in these kinds of laptops got more powerful but the devices have also got lighter than their predecessor. Today laptops can weigh as little as 1 kilo and yet are able to function hundreds of times more efficiently compared to the laptops that were introduced 10 years ago. It is well understood that technology improves with time, but what exactly is the technology involved? Just like our brain functions with the help of biological cells known as neurons, electronic devices function in the same way with help of transistors. The processing unit of any computer or laptop contains billions of transistors. For the Apple Macbook, weighing around 1 kilo, to comprise such a large number of transistors, each transistor must have dimensions of about $500 \mathrm{~nm}$. How is it possible to create anything that is a hundred thousand times smaller than a strand of human hair? Nature produces biological elements from entities as tiny as a bacterium up to huge ones like elephants. But transistors are man-made and it is simply not possible to make nanoscaled objects with hammers or drills.

The term nano-fabrication comes into play. Creation of miniscule objects like nano circuits requires nano-fabrication processes for use in semiconductor device fabrication. Semiconducting materials are the foundation of modern electronics and the most widely used is silicon, as it is easy to work with (for both chemists and physicists) and is also abundantly available in nature, hence it is cheaper than other semiconductor materials. These processes rely on the following advanced integrated circuits fabrication methods: (a) selection of an appropriate base material (substrate); (b) patterning of the substrates by lithography and (c) modifying the electrical properties of the substrate. By the end of this process, devices can be ready for use in applications. In this article insights of a lithography technique used in nano-fabrication shall be touched upon. 


\section{Nanolithography}

The term is derived from Greek words 'nanos' which means dwarf; 'lithos', meaning a stone or rock and 'grapho', which means to write. Various nanolithography tools are ebeam lithography, nano-imprint lithography (NIL), photolithography, X-ray lithography, ion-beam lithography, etc. As their names suggest they use different sources of energy for fashioning designs. Amongst these techniques, photolithography and e-beam lithography techniques are the most common and predominant techniques for nanopatterning in the semiconductor industry.

Nicéphore Niépce, the inventor of photography, first recognized the technique of lithography in 1882. He created patterns by using stones and asphalt in lavender oil on greased paper and left it under the sunlight for two to three hours. The areas that were shielded from the sun remained softer than the unshielded and were easily washed off with lavender oil, leaving behind a pattern formed by the shaded areas. Thus, the first ever photolithography process was carried out.

Modern photolithography is extensively used in industries and has a similar patterning procedure of creating the desired geometries by exposing thin light-sensitive films called 'photoresist' on the substrates and then selectively removing portions of the thin films in developer solvents. Resists are polymeric substances that are formulated to be sensitive to UV light; yes, even sun can do the trick! Stencils or photo-masks are made up of a flat glass area (un-shielding part) and a metal area (shielding counterpart). This mask is kept in direct contact or in close proximity with the substrate coated with photoresists and exposed to UV radiation of varying intensity and subsequently developed in appropriate solvents. A 1:1 image of the entire mask is obtained on the substrate in this manner. Dimensions as small as 70-100 nm can be effortlessly achieved by photolithography.

The consumer market, however, demands faster, more efficient and advanced MacBooks. One way to improve the efficiency is to increase the number of transistors by shrinking their size. Due to the 70-100 nm resolution limit of photolithography other techniques are being developed and electron beam lithography is one of them. Electron beam lithography (EBL) is one of the most favoured tools for patterning processes since structures as small as $7 \mathrm{~nm}$ have been demonstrated. Due to the ease of operation and readily attainable sub-50 nm features, this tool is extensively used in R\&D centres. Moreover, since it is cheaper than setting up optical lithography with similar resolution, it is widely available in universities as well. 


\section{Electron Beam Lithography}

As the name suggests, the EBL system uses a beam of electrons to penetrate through the atoms and molecules to create patterns on different materials. Just like a laser pointer, a highly focused beam of electrons that has a spot size of about $2 \mathrm{~nm}$ (normal laser pointer bears a spot size of 2-3 $\mathrm{mm}$ !) is projected on the resists creating the desired pattern. This is similar to drawing on a paper with a pen. EBL resists are a thin polymer layer deposited on the substrate prior to EBL. It is a sacrificial layer and acts as a stencil to craft the underneath substrate in further processes. EBL resists are especially formulated to respond to electrons. The electrons directly radiate a pattern on resists on a substrate making this techniques mask-less lithography. The electrons locally alternate the chemistry of the resists; either by forming bonds between the resist molecules (negative resist) or cleaving the bonds (positive resists) to make it insoluble (negative resist) or soluble (positive resists) in the appropriate developer. Polymethyl methacrylate, which is also used in constructing glass-substituted aquariums, is a well-established positive EBL resist. It is offered in a liquid state and is spun on the substrates to produce thin films ranging from nanometers to micrometers. Following the exposure to the electron beam, the resist is developed to create the stencil. The design on the resist is, thereafter, engraved through the stencil into the substrate by further processes such as wet chemical etching or dry etching involving gases such as chlorine gas. The process of the development of a negative and positive resist is illustrated in figure 1.
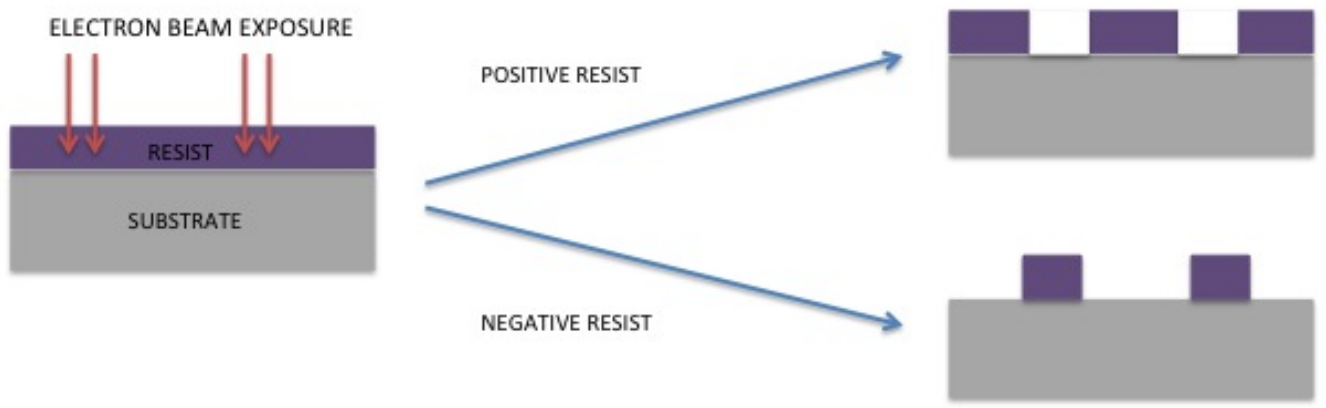

Figure 1: Schematic illustration of development of positive and negative resists after electron beam exposure. Image: Anushka Gangnaik 


\section{What is the study about?}

\section{Aim and study so far}

The main motive of the study is utilising EBL to fabricate novel nano wire devices. As mentioned before, silicon remains the most common substrate to work with. As it is approaching its full potential, however, new materials are being considered as possible alternatives. Germanium has interested the semiconductor industry due to its superior capabilities for the last few years. For instance, germanium has electron mobility twice that of silicon, which simply means that a wire made of germanium will conduct current two times faster than a silicon wire. However with the good comes the bad, germanium is not easy to process due to its 'weird' surface chemistry and hence EBL patterning of such surfaces can be tricky. Germanium surface always needs to be chemically modified thus increasing the time and costs too. The potential of germanium remains unrealised to some extent due to its drawbacks and hence it is the substrate of choice for this study to explore.

Resistors and transistors are very similar in operations and they are ubiquitous components of all electronic circuits. They both regulate the flow of electric current. In the case of transistors, imagine a water pipe with a controlling tap. When the tap is closed we have no water flowing through it. However, the flow of water can be regulated by turning the tap to the required extent. An external force is required here to adjust the water flow. Thus, in transistors, a current (water) flows across the nanowires (pipe) and it is controlled by a gate (tap), which usually sits over the nanowires. On the other hand, resistors are gate-less transistors. The current flow in the resistors can be controlled by adding impurities to the nanowires. For example, another water pipe is stuffed with rocks at certain junctions of the pipe. When the water flows through this pipe, the rocks will hinder the flow of the water. Thus, impurities are introduced into the nanowires to regulate the current flow in a similar fashion. Some impurities in an electronic system are added to speed up current flow. Now, why do we need to regulate current? At home, we can plug a huge appliance like fridge into the same socket where we plug our mobiles. Both the devices, obviously, require different current inputs due to their size and function. Thus, in such a case, the transistors and resistors can help in controlling the flow of electricity through the devices.

This project involves fabricating resistors on a nanometre range on germanium substrates. The study includes measurement of resistance across nanowires of different widths. The size of the wire ranges from $1 \mu \mathrm{m}$ down to $20 \mathrm{~nm}$. There is a need to reduce the resistance and this is done by implanting the nanowires with impurities so that the maximum amount of electricity can pass through the wire and eventually through the whole circuit. This fabrication involves the use of negative resist known as hydrogen silsesquioxane to pattern germanium substrates. 

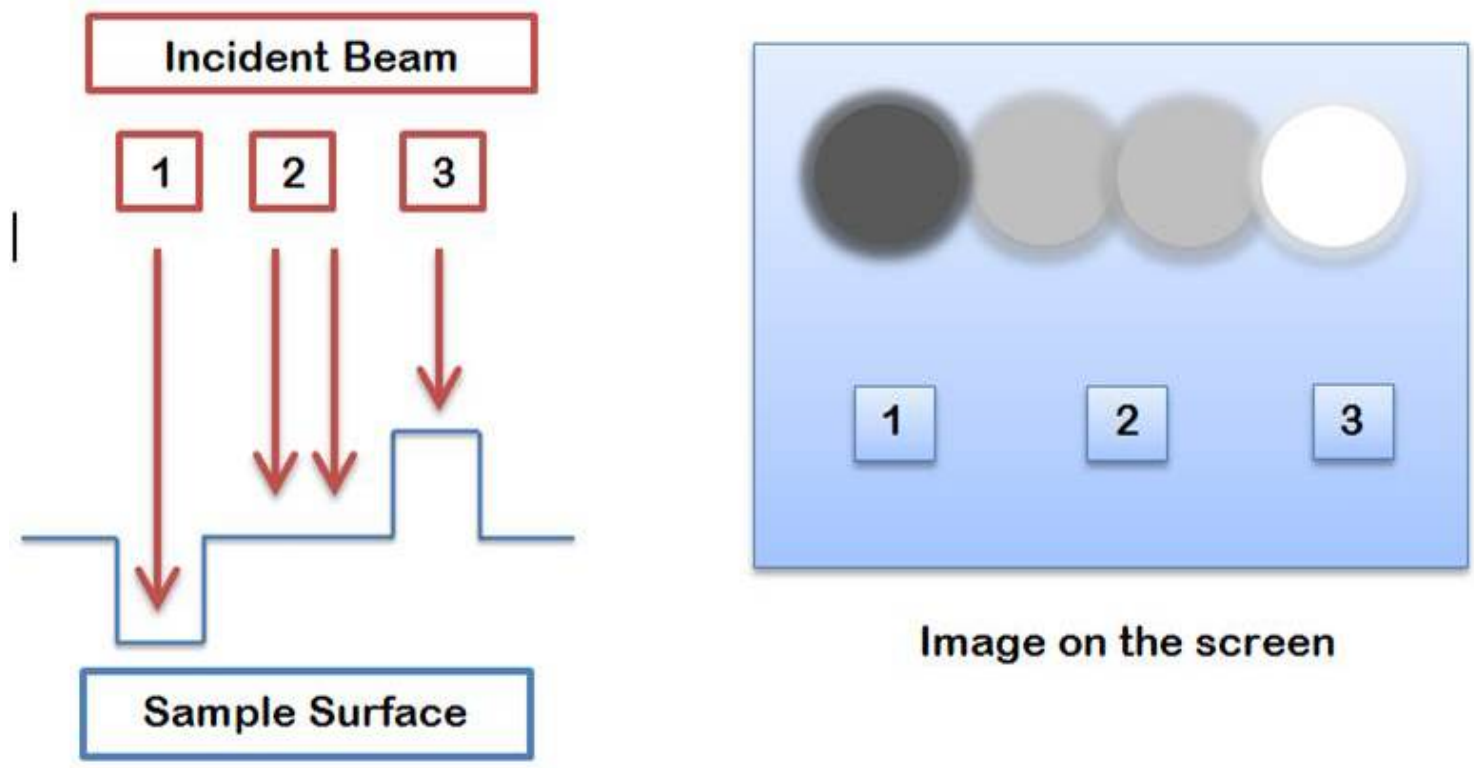

Image on the screen

Figure 2: Illustration of image formation on EBL system. Image: Anushka Gangnaik

The current study also encompasses fabricating nanowires with positive resists known as SML and ZEP. ZEP is a well-established positive tone EBL resist that has demonstrated high-resolution lithography, i.e. capable of producing extremely fine structures. SML, on the other hand, is also a positive tone resist, which is new in the EBL industry and our studies demonstrate that this resist is suitable for EBL.

Nanowires are expected to have many applications in electronics. They are considered to be the main building blocks of the next generation transistors and reducing their dimensions has proved to improve transistor performance. Moreover, miniaturisation of the electronic components will also lead to lighter and smaller devices.

\section{Imaging}

Most EBL systems are not only used for patterning but also for imaging the tiny structures. When the same beam of electrons interacts with the substrate it kicks out some of the substrate-electrons. These electrons are called secondary electrons and are collected by a detector which generates highly magnified images of the nano-scale objects. The contrast in the image is a result of the depths on the surface and is illustrated in figure 2.

It can be seen in figure 2 that when the electron beam scans a surface, the trench will give out less secondary electrons due to the depression and appear darker in the image. Whereas, when it scans over the hill, the maximum amount of secondary electrons will be emitted and then collected by the detector and hence this area would appear very bright. In the intermediate region, from where moderate amounts of electrons will be given out, light grey areas can be observed. In this manner, when the electron beam hits the surface 

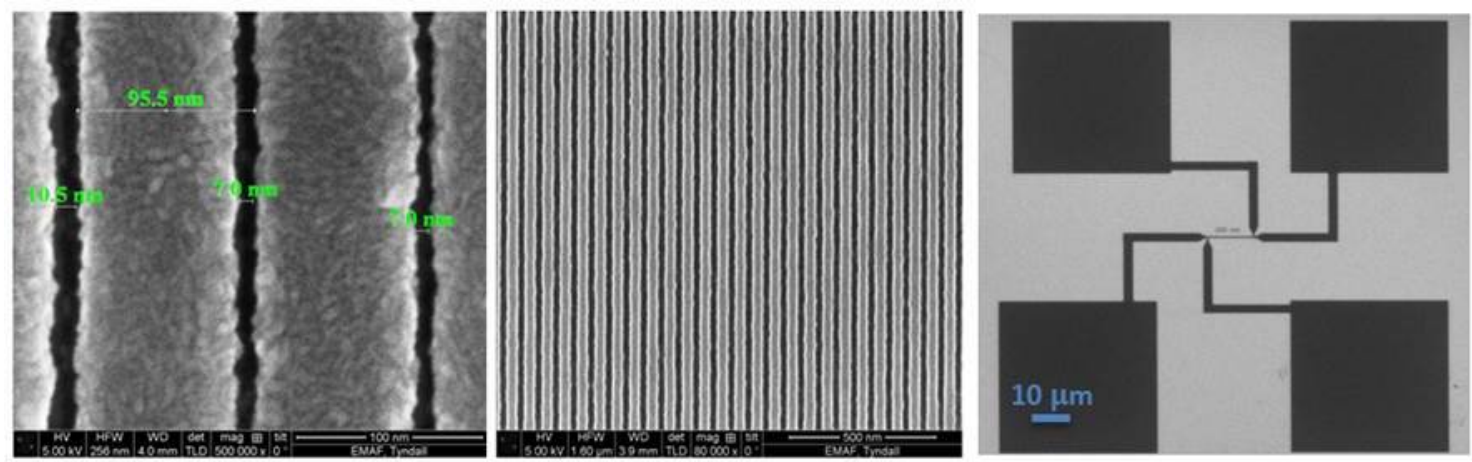

Figure 3: 7-10 nm trenches in ZEP resist, dense silicon nanowires and germanium resistor (from left to right). Image: Anushka Gangnaik

an enormous amount of electrons are removed from the surface which aid in generating complete images. Figure 3 demonstrates the EBL images of trenches on ZEP resist which appear darker due to the depression. The next image is of dense silicon nanowires, since to produce nano devices compaction of space is of utmost importance. The last image is of a germanium resistor, created using EBL. It is used to study current flow through a single nanowire that will be infused with impurities to change its conductance and hence the current flow through it.

\section{Conclusion}

EBL is thus a versatile tool that is user-freindly and can create critically small nanopatterns with lesser efforts. Nanopatterning is required to fulfil the desire for more powerful Apple MacBooks and suchlike devices. Such gadgets will be time and cost effective due to more powerful performance as a result of nano-engineering. This article gives an understanding of nanoscale fabrication that is carried out in the university with the EBL technique and its potential applications.

Anushka Gangnaik is a PhD student in the Chemistry department under the supervision of Prof. Justin D. Holmes. She would like to thank her supervisor and Dr. Yordan Georgiev for their kind guidance. Acknowledgement to Science Foundation Ireland (grant agreement no. 09-IN1-I2602) for funding this ambitious project. 